\title{
Spin Decoherence in Electron Storage Rings *
}

\author{
D.P. Barber, M. Böge, K. Heinemann, H. Mais and G. Ripken \\ Deutsches Elektronen Synchrotron, DESY, \\ 22603 Hamburg, Germany
}

\begin{abstract}
A simple model of spin decoherence in electron storage rings is presented and its relevance to rf spin flipping at high energy is discussed.
\end{abstract}

\section{INTRODUCTION}

Stored electron and positron beams can become spin polarized by the emission of synchrotron polarization - the so called Sokolov-Ternov effect $(1,2,3)$. In rings without vertical bends and solenoids, the polarization is vertical, antiparallel to the guide field. It has recently been demonstrated at HERA that spin rotators can be used to rotate the polarization vector into the beam direction just before an interaction point and back again after the interaction point so that longitudinally polarized electrons or positrons are available for the high energy physics experiment(4).

Periodic reversal of the helicity is essential for the physics programme and it is clear that it would also be useful to have a means of flipping the polarization direction for short periods in order to check for systematic errors. The helicity at the interaction point can be reversed by changing the geometry and fields of the rotators but that would mean a temporary loss of polarization or even dumping the beam and refilling. However, a faster, more convenient method was already considered many years ago $(5,6)$ and would utilize a horizontal rf magnetic field.

\footnotetext{
${ }^{*}$ Expanded and updated version of a talk given at the 11th International Symposium on
} High Energy Spin Physics, Bloomington, Indiana, USA, September 1994. 
The rf magnetic field (or a combination of fields forming a closed bump) $(5,7)$ would be installed at a position on the ring where the polarization were vertical and it would run in resonance with the natural spin precession frequency i.e. at a frequency close to $f_{\text {flip }}=f_{c} \cdot \tilde{\nu}$ or $f_{\text {flip }}=f_{c} \cdot(1-\tilde{\nu})$ where $f_{c}$ is the circulation frequency and $\tilde{\nu}$ is the fractional part of the spin tune, $\nu$, which is the number of spin precessions per turn around the ring (19).

Flipping would involve sweeping slowly enough across resonance to ensure that the polarization vector were tipped over adiabatically. This would require that the spins in a bunch remain tightly bundled. Such flipping techniques are routine at Novosibirsk $(8,9)$ at low energy. These techniques are very closely related to the method used to depolarize a beam and hence measure its energy by noting the required $\mathrm{rf}$ frequency $(10,11,12){ }^{1}$

It has been suggested that if flipping were repeated at the suitable intervals it would perhaps be possible to reach a periodic limit cycle for the polarization $(13,14,15)$.

However, if the projections of the spins on the horizontal plane were to become spread out uniformly over the range $\pm \pi$ (in an appropriate coordinate system) during the sweep process, i.e. if there were complete decoherence, the polarization vector would not be flipped but instead the polarization would vanish. As we will see, one such source of decoherence is the stochastic nature of synchrotron radiation photon emission. In proton rings, decoherence of this nature cannot occur and full spin flip is not difficult to achieve (16).

Spin flip was sometimes observed at LEP during energy calibrations (12) using rf fields of just a few gauss-metres but the value of the polarization was much reduced and the effect was not consistently reproducible. It is also unclear which are the best ranges of sweep rate and rf field strength (17).

But the fact that flip can be achieved suggests that the spin projections remain coherent at least for several seconds during the sweep. Thus in order to better understand the measurements it would be useful to estimate the decoherence rate. One such calculation suggests that the characteristic decoherence time is proportional to the fourth power of the synchrotron tune and could indeed be several minutes at LEP (18).

In this article we show, by a more complete treatment of the photon emission process and the subsequent development of the spin distribution function, that with the same linear "smooth ring" model for the synchrotron motion as in reference 18, the spin distribution actually reaches equilibrium in a few damping times and that there need not be full decoherence. We then consider other sources of decoherence and their consequences.

The calculation presented below is a very abbreviated version of a full treatment based on a well defined and trusted formalism. The full calculation

\footnotetext{
${ }^{1}$ Actually it is the spin tune extracted from the complex eigenvalues of the one turn $3 \mathrm{x}$ 3 spin transport matrix that is measured (19).
} 
will be published elsewhere.

\section{EQUATIONS OF LINEARIZED ORBIT MOTION}

The linearized equation of orbit motion with respect to the closed orbit in the presence of stochastic excitation and damping due to synchrotron radiation takes the form used in the SLIM program (19):

$$
\frac{d}{d s} \overrightarrow{\hat{y}}=\underline{\hat{A}} \cdot \overrightarrow{\hat{y}}+\delta \underline{\hat{A}} \cdot \overrightarrow{\hat{y}}+\delta \overrightarrow{\hat{c}}
$$

where $s$ is the distance around the ring and $\overrightarrow{\hat{y}}$ is the vector of orbit variables $\left(\hat{x}, \hat{p}_{x}, \hat{z}, \hat{p}_{z}, \hat{\sigma}, \hat{p}_{\sigma}\right)$. Here, $\hat{\sigma}$ is the distance to the centre of the bunch and $\hat{p}_{\sigma}$ is the fractional energy deviation. $\underline{\hat{A}}$ represents the 'hamiltonian' motion due to the Lorentz forces and $\delta \underline{\hat{A}}$ describes damping. Both are $s$-dependent $6 \times 6$ matrices. The vector $\delta \overrightarrow{\hat{c}}=(0,0,0,0,0, \delta c)$ accounts for the stochastic excitation in the energy variable due to photon emission (20):

$$
\delta c=\sqrt{\omega} \cdot \xi(s),
$$

where in terms of the curvatures $K_{x}$ and $K_{z}, \omega=\left(\left|K_{x}\right|^{3}+\left|K_{z}\right|^{3}\right) \cdot C_{2}$ with

$$
C_{2}=\frac{55 \cdot \sqrt{3}}{48} \cdot C_{1} \cdot \Lambda \cdot \gamma_{0}^{2} ; \quad C_{1}=\frac{2}{3} e^{2} \cdot \frac{\gamma_{0}^{4}}{E_{0}} ; \quad \Lambda=\frac{\hbar}{m_{0} c}
$$

and where the stochastic averages of the kicks $\xi(s)$ are

$$
<\xi(s) \cdot \xi\left(s^{\prime}\right)>=\delta\left(s-s^{\prime}\right) ; \quad<\xi(s)>=0 .
$$

Thus, as is usual and sufficient $(20,21,22)$, we take the synchrotron radiation to be a white noise process. For our present purpose it will be more convenient to work with dynamical variables which allow a clearer separation of the influence of energy oscillations from the purely "betatron" motion due to the quadrupoles. To achieve this we introduce the dispersion by means of a canonical transformation to obtain a new set of variables $\left(x, p_{x}, z, p_{z}, \sigma, p_{\sigma}\right)$ defined by:

$$
\begin{aligned}
x & =\hat{x}-\hat{p}_{\sigma} \cdot D_{x} ; \\
p_{x} & =\hat{p}_{x}-\hat{p}_{\sigma} \cdot D_{x}^{\prime} ; \\
z & =\hat{z}-\hat{p}_{\sigma} \cdot D_{z} ; \\
p_{z} & =\hat{p}_{z}-\hat{p}_{\sigma} \cdot D_{z}^{\prime} \\
\sigma & =\hat{\sigma}-\hat{p}_{x} \cdot D_{x}+\hat{x} \cdot D_{x}^{\prime}-\hat{p}_{z} \cdot D_{z}+\hat{z} \cdot D_{z}^{\prime} ; \\
p_{\sigma} & =\hat{p}_{\sigma}
\end{aligned}
$$


where the $D^{\prime} s$ are the components of the dispersion vector (23).

In terms of the variables $x, p_{x}, z, p_{z}, \sigma, p_{\sigma}$ the equation of motion now takes the form:

$$
\frac{d}{d s} \vec{y}=\underline{A} \cdot \vec{y}+\delta \underline{A} \cdot \vec{y}+\delta \vec{c} .
$$

If the dispersion is zero at the position of the rf cavities, there is no transverselongitudinal coupling and the matrices $\underline{A}$ and $\delta \underline{A}$ have a simple block diagonal form. For example:

$$
\underline{A}(s)=\left(\begin{array}{cc}
\underline{A}_{(4 \times 4)}^{(\beta)}(s) & \underline{0}_{(4 \times 2)}^{(\sigma)} \\
\underline{0}_{(2 \times 4)} & \underline{A}_{(2 \times 2)}^{(s)}
\end{array}\right) .
$$

The matrix $\underline{A}_{(4 \times 4)}^{(\beta)}(s)$ describes betatron motion in the focussing fields. $\underline{A}_{(2 \times 2)}^{(\sigma)}(s)$ describes the synchrotron motion. When acting alone this gives :

$$
\begin{aligned}
\frac{d}{d s} \sigma & =-\left[K_{x} \cdot D_{x}+K_{z} \cdot D_{z}\right] \cdot p_{\sigma} ; \\
\frac{d}{d s} p_{\sigma} & =h \cdot \frac{2 \pi}{L} \cdot \frac{e V(s)}{E_{0}} \cos \varphi \cdot \sigma,
\end{aligned}
$$

where the symbols have their usual meanings.

In this calculation we also work in the "smooth ring" approximation and consider only synchrotron motion. Thus we will follow exactly the philosophy of reference 18 . So the matrix elements in $\underline{A}_{(2 \times 2)}^{(\sigma)}$ and $\delta \underline{A}_{(2 \times 2)}^{(\sigma)}$ are averaged over one turn (of length $L$ ) and we obtain :

$$
\left(\begin{array}{c}
\sigma^{\prime} \\
p_{\sigma}^{\prime}
\end{array}\right)=\left(\begin{array}{cc}
0 & -\kappa \\
\Omega_{s}^{2} / \kappa & 0
\end{array}\right) \cdot\left(\begin{array}{c}
\sigma \\
p_{\sigma}
\end{array}\right)+\delta \underline{A}_{(2 \times 2)}^{(\sigma)} \cdot\left(\begin{array}{c}
\sigma \\
p_{\sigma}
\end{array}\right)+\delta \vec{c},
$$

where $\delta \underline{A}_{(2 \times 2)}^{(\sigma)}$ and $\delta \vec{c}$ take the forms:

$$
\delta \underline{A}_{(2 \times 2)}^{(\sigma)} \equiv\left(\begin{array}{cc}
0 & 0 \\
0 & -2 \cdot \alpha_{s} / L
\end{array}\right), \quad \delta \vec{c} \equiv \sqrt{\tilde{\omega}} \cdot\left(\begin{array}{c}
0 \\
\xi(s)
\end{array}\right) .
$$

Here, $\alpha_{s}$ is the one turn synchrotron damping decrement and $\tilde{\omega}$ is the one turn averaged $\omega$. Also, $\Omega_{s}=2 \pi \cdot Q_{s} / L$ where $Q_{s}$ is the synchrotron tune and $\kappa$ is the compaction factor.

The equilibrium covariance matrix for $\sigma$ and $p_{\sigma}$ then takes the usual value (24) viz :

$$
\underline{\sigma}_{2}(\infty)=\left(\begin{array}{cc}
\sigma_{\sigma}^{2} & 0 \\
0 & \sigma_{p_{\sigma}}^{2}
\end{array}\right), \quad \sigma_{p_{\sigma}}^{2}=\frac{\tilde{\omega} \cdot L}{4 \cdot \alpha_{s}}, \quad \sigma_{\sigma}^{2}=\frac{\kappa^{2}}{\Omega_{s}^{2}} \cdot \sigma_{p_{\sigma}}^{2} .
$$




\section{INCLUSION OF SPIN}

After this recapitulation of the basis for the matrix formulation of the standard smoothed description of damped stochastic synchrotron motion we are in a position to introduce spin motion. Although spin is a quantum mechanical phenomenon, in high energy storage rings it can be treated at the semiclassical level using the Thomas-BMT equation (19)

$$
\frac{d}{d s} \vec{S}=\vec{\Omega} \times \vec{S}
$$

describing the precession of a classical spin $\vec{S}$ in electric and magnetic fields. The precession vector $\vec{\Omega}$ is a function of the magnetic and electric fields and of the particle velocity and energy. As is usual for spin calculations in storage rings we now write $\vec{\Omega}$ as a sum of a piece $\vec{\Omega}_{0}$ accounting for the fields on the closed orbit and a piece $\vec{\Omega}_{\text {osc }}$ accounting for synchro-betatron motion with respect to the closed orbit.

We will assume that the ring has no vertical bends, solenoids or skew quadrupoles, and that it is perfectly aligned so that there is no vertical closed orbit deviation. For electrons the vertical emittance can then be taken to be zero and only motion in the horizontal plane need be considered. For this naive estimate the betatron motion and the radial rf magnetic field will be ignored. Spin motion will be calculated with respect to a pair of mutually orthogonal axes precessing at the rate $\Omega_{0}$ in the horizontal plane around the vertical dipole field. The direction of a horizontal spin in this frame is denoted by a phase angle $\psi$ so that we have $\psi^{\prime}=\Omega_{o s c}$. After averaging we then obtain $\psi^{\prime}=2 \pi \nu / L \cdot p_{\sigma}$ where the spin tune $\nu$ is $(g-2) / 2 \cdot \gamma$. Thus $\psi$ only couples to and is only driven by $p_{\sigma}$. When the spin phase $\psi$ is included, the stochastic differential equation for the system takes the form :

$$
\left(\begin{array}{c}
\sigma^{\prime} \\
p_{\sigma}^{\prime} \\
\psi^{\prime}
\end{array}\right)=\underbrace{\left(\begin{array}{ccc}
0 & a & 0 \\
b & 0 & 0 \\
0 & d & 0
\end{array}\right) \cdot\left(\begin{array}{c}
\sigma \\
p_{\sigma} \\
\psi
\end{array}\right)}_{\text {Hamiltonian motion }}+\underbrace{\left(\begin{array}{ccc}
0 & 0 & 0 \\
0 & c & 0 \\
0 & 0 & 0
\end{array}\right) \cdot\left(\begin{array}{c}
\sigma \\
p_{\sigma} \\
\psi
\end{array}\right)}_{\text {Damping }}+\underbrace{\sqrt{\tilde{\omega}} \cdot\left(\begin{array}{l}
0 \\
\xi \\
0
\end{array}\right)}_{\text {Excitation }},
$$

where the constants $a, b, c$ and $d$ are defined as :

$$
a=-\kappa, b=\Omega_{s}^{2} / \kappa, c=-2 \cdot \alpha_{s} / L, d=2 \pi \nu / L .
$$

This can be rewritten in the form:

$$
\vec{x}^{\prime}=\underline{\mathcal{A}} \cdot \vec{x}+\delta \vec{c}_{3}
$$


where

$$
\vec{x} \equiv\left(\begin{array}{c}
\sigma \\
p_{\sigma} \\
\psi
\end{array}\right), \quad \underline{\mathcal{A}} \equiv\left(\begin{array}{ccc}
0 & a & 0 \\
b & c & 0 \\
0 & d & 0
\end{array}\right), \quad \delta \vec{c}_{3} \equiv \sqrt{\tilde{\omega}} \cdot\left(\begin{array}{c}
0 \\
\xi \\
0
\end{array}\right) .
$$

This linear Langevin equation is interpreted according to the Stratonovich convention and leads to the following Fokker-Planck equation $(25,26)$ for the distribution function $W\left(\sigma, p_{\sigma}, \psi\right)$ :

$$
\frac{\partial W}{\partial s}=-\sum_{j=1}^{3} \frac{\partial}{\partial x_{j}}\left[\mathcal{D}_{j} \cdot W\right]+\sum_{i, j=1}^{3} \frac{\partial^{2}}{\partial x_{i} \partial x_{j}}\left[\mathcal{D}_{i j} \cdot W\right],
$$

where

$$
\mathcal{D}_{j} \equiv \sum_{k=1}^{3} \mathcal{A}_{j k} \cdot x_{k}, \quad \mathcal{D}_{i j} \equiv \frac{\tilde{\omega}}{2} \cdot \delta_{i j} \cdot \delta_{i 2} \quad(i, j=1,2,3) .
$$

So the Fokker-Planck equation has the final form :

$$
\begin{aligned}
\frac{\partial W}{\partial s}=-c \cdot & W-a \cdot p_{\sigma} \cdot \frac{\partial W}{\partial \sigma}-\left[b \cdot \sigma+c \cdot p_{\sigma}\right] \cdot \frac{\partial W}{\partial p_{\sigma}}-d \cdot p_{\sigma} \cdot \frac{\partial W}{\partial \psi} \\
+ & \frac{\tilde{\omega}}{2} \cdot \frac{\partial^{2} W}{\partial p_{\sigma}{ }^{2}} .
\end{aligned}
$$

With such Fokker-Planck formulations for this and more complicated models we can carry out perfectly standard detailed studies of spin decoherence under all possible conditions just by looking for the possible solutions for $W\left(\sigma, p_{\sigma}, \psi\right)$ compatible with the initial conditions. In the present model, by starting with a delta function distribution in $\sigma, p_{\sigma}$ and $\psi$, corresponding to a pointlike beam and a tight bundle of spin projections, the distribution function (i.e. the transition probability in this case ) evolves so that the covariance matrix for the $\sigma, p_{\sigma}$ and $\psi$ is given by $(25,26)$ :

$$
\underline{\sigma}_{3}(s)=2 \cdot \int_{0}^{s} d s^{\prime} \underline{M}\left(s^{\prime}\right) \cdot \underline{\mathcal{D}} \cdot \underline{M}^{T}\left(s^{\prime}\right),
$$

where $M$ is the real valued transfer matrix solving :

$$
\underline{M}^{\prime}=\underline{\mathcal{A}} \cdot \underline{M}, \quad \underline{M}(s=0)=\underline{1} .
$$

After some initial damped oscillatory behaviour, in a few synchrotron damping times the elements of $\underline{\sigma}_{3}$ reach the asymptotic values :

$$
\underline{\sigma}_{3}(\infty)=\left(\begin{array}{ccc}
\sigma_{\sigma}^{2} & 0 & \frac{d}{a} \cdot \sigma_{\sigma}^{2} \\
0 & \sigma_{p_{\sigma}}^{2} & 0 \\
\frac{d}{a} \cdot \sigma_{\sigma}^{2} & 0 & \frac{d^{2}}{a^{2}} \cdot \sigma_{\sigma}^{2}
\end{array}\right)
$$


This result follows exactly from the stochastic differential equation 19. Thus the $\sigma$ and $p_{\sigma}$ distributions acquire the equilibrium spreads given earlier. This is to be expected since in these approximations the spin has no influence on the orbital motion. However, and this is perhaps unexpected, equation 28 shows that the $\psi$ distribution also reaches equilibrium (on the same time scale) with a value $\sigma_{\psi}(\infty)=\left|\frac{d}{a}\right| \cdot \sigma_{\sigma}=\nu \sigma_{p_{\sigma}} / Q_{s}$ : apart from an initial decoherence lasting a few dampimg times there is no continual decoherence in this model with these starting conditions! But of course, if $\sigma_{\psi}(\infty)$ were very large the spins would be effectively decoherent.

In the HERA electron ring at $27.5 \mathrm{GeV}, \nu$ is about $62.5, \sigma_{p_{\sigma}}$ is about $10^{-3}$ and $Q_{s}$ is about 0.06 . So the asymptotic $\sigma_{\psi}$ is about 60 degrees corresponding to a polarization of about $58 \%$. However, several extra points should be noted. Firstly, $\psi$ is correlated to $\sigma$, not as one might have expected, to the energy deviation $p_{\sigma}$. Secondly, the last column of $\underline{\mathcal{A}}$ is empty and the $\underline{\sigma}_{3}(s)$ is singular for all $s$. For a linear problem such as this, one expects that the asymptotic $W$ is a generalized gaussian in $\sigma, p_{\sigma}$ and $\psi$. But, the coefficients of the quadratic form in the exponent of this gaussian clearly cannot be obtained by inverting $\underline{\sigma}_{3}(s)$. So another method suitable for problems of this type must be used. Then one finds that the asymptotic $W$ function is not unique but reaches an equilibrium form depending on the initial conditions. For example to discuss decoherence according to the picture in the Introduction, one begins with gaussian distributions in $\sigma$ and $p_{\sigma}$ with their equilibrium asymptotic variances and with a delta function distribution $\delta(\psi)$ in $\psi$. Then the asymptotic $\psi$ distribution has a variance of $\sqrt{2}\left(\nu \sigma_{p_{\sigma}} / Q_{s}\right)$ which is about 85 degrees. So far we have allowed the azimuthal angle $\psi$ to cover the range $\pm \infty$ whereas the physical range is $\pm \pi$. If this latter is taken into account the asymptotic polarization in this case is about $34 \%^{2}$. This calculation and further aspects of the problem will be treated in another article (27). The asymptotic variances of $\sigma$ and $p_{\sigma}$ are unique.

According to our model, in machines running at one or two $\mathrm{GeV}$, the asymptotic $\sigma_{\psi}$ is just a few degrees. So within this simple linear model there is no complete decoherence in such machines. Conventional wisdom suggests instead that $\sigma_{\psi}$ should increase as $\sqrt{s}$. This is not the case as we have just seen. However, in the simpler $2 \times 2$ pure diffusion problem for $p_{\sigma}$ and $\psi$ without synchrotron oscillations the $\sqrt{s}$ growth does emerge after a few damping times and for HERA quickly results in complete decoherence. So the synchrotron motion is an essential ingredient in our calculation.

So far we have neglected the detailed structure of the ring and misalignments which tilt the equilibrium polarization axis and generate vertical dispersion. Horizontal and vertical betatron motion have been neglected as have the

\footnotetext{
${ }^{2}$ This effect was also taken into account when calculating the $58 \%$ mentioned above.
} 
nonlinear spin tune spread and the effects of sextupoles and spin rotators. So our model is perhaps too simple to represent a realistic storage ring but it has enabled us to reconsider the calculation in reference 18. If a Siberian Snake is included in the otherwise smoothed ring there is decoherence (27).

By considering decoherence in isolation, a key component, the rf field itself, was ignored. Recall, however, that partial spin flip was sometimes seen at LEP with small rf fields. This suggests that the predictions of the model are not too unrealistic.

\section{ACKNOWLEDGMENTS}

We wish to thank J-P. Koutchouk, B. Dehning and Yu. Shatunov for useful discussions.

\section{REFERENCES}

1. Sokolov, A.A. and Ternov, I.M.,Sov. Phys. Doklady, 8, 1203 (1964).

2. Barber, D.P.,Proc.9th Int.Symp.High Energy Spin Physics, Bonn,Germany,1990.

3. Barber, D.P., Proc.2nd Euro.Part.Acc.Conf,, Nice, France, 1990.

4. Barber, D.P., et al., DESY Report 94-171 (1994). To appear in Phys.Letts. B.

5. Schwitters, R., DESY Report M-82-09 (1982).

6. Shatunov, Yu., DESY Report M-82-09 (1982).

7. Barber, D.P., Discussion session at DESY "Harz Seminar":February 1994.

8. Vasserman, I.B., et al., Phys. Letts., B198, 302 (1987) and references therein.

9. Shatunov, Yu., These Proceedings.

10. Barber, D.P., et al., Phys. Letts., B135, 498 (1984).

11. Grosshauser, C., Diplom thesis, DESY-HERMES Report 94-01 (1994).

12. Dehning, B., These Proceedings and references therein.

.... Arnaudon,L., et al., CERN Report SL/94-71 (1994) and references therein.

13. Jowett, J.M. and Ruth, R.D., CERN Report LEP Th/83-26 (1983).

14. Yokoya, K., Particle Accelerators, 14, 39 (1983).

15. Düren, M., DESY Report DESY HERA 94-03 (1994).

16. See for example:

.... Huang, H., et al., Phys. Rev. Letts., 73, 2982 (1994) and referencies therein.

17. Dehning, B., Private communication.

18. Koutchouk, J.P., CERN Note SL/AP-16 (1991).

19. Chao, A.W., Nucl. Inst. Meth., 180, 29 (1981).

20. Barber, D.P., et al., DESY Report 91-146 (1991).

21. Jowett, J.M., AIP Conference Proceedings, 153, 1987.

22. Ruggiero, F., et al., Annals of Physics 197, 396 (1990).

23. Barber, D.P., et al., DESY Report HERA 94-02 (1994).

24. Mais, H. and Ripken,G., DESY Report 86-29 (1986).

25. Risken, H., The Fokker-Planck Equation, 2nd edition, Springer 1989. 
26. Gardiner, G.W., Handbook of Stochastic Methods, 2nd edition, Springer 1985.

27. K. Heinemann, DESY Report 97-166 (1997), arXiv:physics/9709025.

28. Böge, M., DESY Report 94-87 (1994). 